\title{
Damage Analysis of Materials with Microvoids
}

\section{DU SHANYI and LIU YINGJIE}

School of Astronautics, Harbin Institute of Technology,

$$
\text { Harbins, PRC }
$$

\section{INTRODUCTION}

Generally practical materials have some microflaws or macroflaws. It is an important to analyze the macroscopic behavior of these materials with Damage Mechanics.

Kachanov (1), Lemaitre and chaboche [2] introduced the effective stress and described the effect of microflaws on the macroscoDic behavior of materials. Rousselier (3) assumed that damade

variable is associated with material density. Mclintock (4), Rice and Tracey ${ }^{5} 5$ analyzed the damace materals with microscopic model. An anisotropic damare variable which has more general sense is given with associative method of macroscopic and microscopic models in this naner. The differential equation of state function for the anisotronic damage with microvoids is qiven and its anpriximate solution is obtained. For the special case of spherical voids, the final result is compared with Rousselier's result. It is also pointed out that plastic potential is associated with the stress state and growth way of microvoids.

ANISOTROPIC DAMASE VARIABLE

Lemaitre ${ }^{(6)}$ cosidered the free eneray function as follows

$$
\psi=\psi_{e} e^{\left(\varepsilon^{e}, D\right)}+\psi_{p}(p)
$$

where $)$ is elastic enercy density associated with damade; "p is the free energy associated with accumulative plastic deformation. We is aiven as

$$
\psi^{\prime}=\frac{1}{2 \rho} \underline{\varepsilon}^{e}: \underline{c}: \underline{\varepsilon} e(1-D)
$$

let $\underline{\widetilde{c}}=\underline{c}(1-D)$, then equation (2) becomes 


$$
\psi_{\mathrm{e}}=\frac{1}{2 \rho} \underline{\varepsilon}^{\mathrm{e}}: \underline{\tilde{\mathrm{c}}}: \underline{\varepsilon}^{\mathrm{e}}
$$

$\widetilde{\widetilde{c}}$ is the effective elastic constant. Damage is the state variable which has an effect on the elastic constant according to equation (3). It is also obtained as

$$
\underline{\sigma}=\underline{\mathrm{C}}:(\underline{\mathrm{I}}-\underline{\mathrm{D}}): \underline{\varepsilon}^{\mathrm{e}}=\underline{\tilde{\mathrm{c}}}: \underline{\varepsilon}^{\mathrm{e}}
$$

The damage variable $\underline{D}$ can be determined from $\underline{\widetilde{c}}$.

Consider an infinite body including the finite inhomogeneous reqion with the ellipsoidal voids of same orientation and the matrix of the body is an isotropic medium as shown in Fig.l.

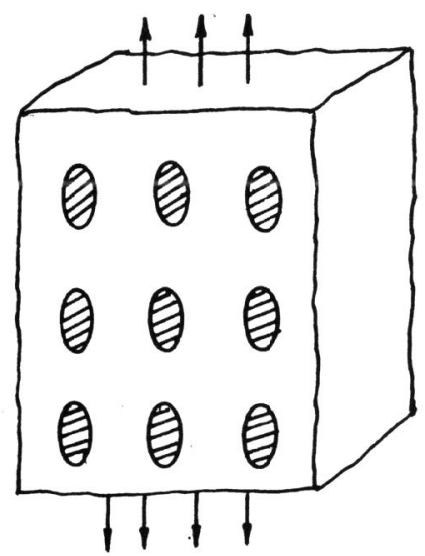

Fig. 1

Refs. (7) and (8) were given the solutions of problems of single and more inclusions, resnectivelv. Let $\Sigma_{i}$.j and $E_{i j}$ be the alobal
stress and strain of the body, then the strain energy is as

$$
W=\frac{1}{2} \tilde{c}_{i, j k 1}^{-1} \Sigma_{i j} \Sigma_{k l}
$$

$\widetilde{c}_{i j k l}$ indicates the effective elastic modulus. It is as

$$
\tilde{C}^{-1}{ }_{i, j k l}=\mathrm{C}^{0-1}{ }_{i, j k l}+f A_{i j m n} C^{0-1} m n k l
$$

$\mathrm{C}^{0}{ }_{i j k l}, f$ and $\mathrm{Aijmn}$ are elastic modulus of matrix, ellipsoidal inclusion volume fraction and cofficient associated with the size and volume fraction of ellipsoidal voilds( $(9)$. In the form

$$
\tilde{\mathrm{C}}^{-1}=\underline{\mathrm{C}}^{-1}+\underline{\mathrm{f}} \underline{\mathrm{A}}: \underline{\mathrm{C}}^{-1}
$$

From equation (4), the anisotronic damage variable is as

$$
\underline{D}=\underline{I}-\underline{C}^{-1}:\left(\underline{C}^{-1}+f \underline{A}: \underline{C}^{-1}\right)^{-1}
$$

It is noticed that the damane variable depends on the volume fraction and size of ellinsoidal voids. So $\underline{D}$ can be assumed as follows

$$
\underline{D}=\underline{D}(\gamma, f)
$$

where $\gamma$ is the ratio between the long and short axis. $D$ can be assumed the simple function of $\gamma$ and $f$ for some special problems.

\section{DIFERENTIAL EQUATION OF THE STATE FUNCTION}

In this paper, the followina assumbtion are made:

a) The flaws are the forms of microvoids after yieldina; b) The a The flaws are the for the general standard material; c) The material with dama is or the general standariable of irredamare parameter is an independent internantinuance conditions.

If the arowth of microvoids is isotropic, damage dissipation of If the growth of microvoids is isotropic, damage dissipation of eneray consists of two parts: the dissipation of eneray formina new surface. Let wg indicate the sum of two parts.

For an infinite homogeneous medium with an ellinsoidal void, Wg is expressed as

$$
W_{g}=\frac{\sigma m}{\rho} \frac{f}{1-f}+c \cdot \dot{s}
$$

where $\sigma_{\mathrm{m}}, r_{3}$ and $S$ are the averaqe stress, surface enercy of unit area and area of microvoids. In fact, $c_{2}<<\sigma m$ in quantity, so

$$
W g=\frac{\sigma m}{\rho} \frac{\dot{f}}{1-f}
$$
The state function can be divided into two parts. One is free
eneray shown in equation ( 3 ). Another one is potential of dissination which can be assumed as

$$
\psi=\psi_{1}(p)+\psi_{2}\left(\gamma, f, \varepsilon_{i j}^{e}\right)
$$

From equation (12), we have

$$
B_{\gamma}(d)=\frac{\partial \psi_{2}}{\partial \gamma}, B_{f}(d)=\frac{\partial \psi_{2}}{\partial f}, A=\frac{\partial \psi_{1}}{\partial p}
$$

Substituting eqn. (13) into (11), one obtains

$$
B_{\gamma}{ }^{(d)} \dot{\gamma}+B_{f}{ }^{(d)} \dot{f}=\frac{\sigma m}{p} \frac{\dot{f}}{1-f}
$$

$\gamma=0$ when the arowth of ellipsoidal voids is isotronic. Then

$$
\frac{\partial \psi_{2}}{\partial f}=\frac{1}{\rho} \sigma m \frac{1}{1-f}=\left(\frac{\partial \psi_{e}}{\partial \varepsilon_{\mathrm{m}}}+\frac{\partial \psi_{2}}{\partial \varepsilon_{\mathrm{m}}^{e}}\right) \frac{1}{3(1-f)}
$$

Accordina to ean. (3), one gets 


$$
\frac{\partial u_{e}}{\partial \varepsilon \tilde{m}_{n}}=\frac{1}{3} \tilde{c}_{m n k l} \varepsilon_{k l}
$$

Let $\widetilde{C}_{\text {mnkl }}=K_{k l}(\gamma, f)$ be known function, take approximate solution

$$
\psi_{2}=f_{1}(\gamma, f)+f_{i j}(\gamma, f) \varepsilon_{i j} e
$$

Substitutina eqn. (17) into (15), one obtains

$$
\frac{\partial f_{i}}{\partial f}=\frac{1}{1-f} f_{i i}(\gamma, f), \frac{\partial f_{i j}}{\partial f}=\frac{1}{(1-f)} k_{i j}(\gamma, f)
$$
In the case of determined $\gamma$ and $f, \psi_{2}$ can be obtained by equ.

\section{PROBLEM OF SPHERICAL VOIDS}

Assume spherical voids does not affect on elastic modulus of material, then

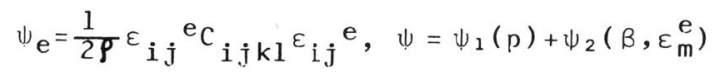

$B$ is damane variable of microvoids. When $\gamma=1$, then

$$
B=B(f)
$$

Accordina to the constitutive relation, we have

$$
\sigma_{i, j}=\frac{\partial \psi_{e}}{\partial \varepsilon_{i j}^{e}}+\frac{\partial \psi_{2}}{\partial \varepsilon_{i j}^{e}}, \quad B=\frac{\partial \Downarrow_{2}}{\partial \beta}, \quad A=\frac{\partial \psi_{1}}{\partial p}
$$

From eqn. (19) and (21), one obtains

$$
\frac{\sigma m}{\rho}=K \varepsilon_{m}^{e}+\frac{1}{3} \frac{\partial \Psi_{2}}{\partial \varepsilon_{m}^{e}}
$$

where $K=E / 3(1-\mu)$. Substitutina ean. (22) into ean. (15), the followina equation is niven

$$
\boldsymbol{\rho}_{\frac{\partial \psi_{2}}{\partial B} \dot{B}}=\left(\frac{1}{3} \frac{\partial \psi_{2}}{\partial \varepsilon_{\mathrm{m}}^{\mathrm{e}}}+K \varepsilon_{\mathrm{m}}^{\mathrm{e}}\right) \frac{1}{1-f}
$$

renerality is not lost, let $B=\operatorname{Ln}(1-f)$, then

$$
\psi_{2}=-\frac{1}{\rho} K B \varepsilon_{m}^{e}+\frac{k}{6} \beta^{2}
$$

Equation of $\psi=\psi_{1}(p)+\|_{2}(\beta)$ is chosen and ean. (14) is not mentioned in Ref. (3).

The discussion of plastic notential is based on semianalytical assumption. It is reasonable that $\psi_{2}$ is function of $\beta$ and $\varepsilon_{m}$.

Take $B=\beta(\rho)$, one obtains

$$
B^{\prime}(\rho) \psi_{2}^{\prime}(\beta)=-\frac{\sigma m}{\rho}
$$

The defference between ern. (26) and result of Ref. [3] is that the rinht term of Ref. (3) is $\sigma_{0} / \mathrm{c}$. In fact, the result of Ref. (3) is the approximate result in the case of perfectly plas-

ticity.

Take plastic potential as ceneral form as

$$
F\left(\frac{\sigma}{\rho}, \psi_{1}(p), \psi_{2}(R), B\right)=\left[J_{2}\left(\frac{\sigma}{\rho}\right)\right]^{\frac{1}{2}}+\frac{\psi_{1}(p)}{3}+F_{1}\left(\psi_{2}, R\right)
$$

The plastic potential of isotronic arowth of snherical voids can be obtained with similar method of Ref. 3 and it is as follows

$$
{ }^{1} F=\left[J_{2}\left(\frac{\sigma}{\rho}\right)\right]^{\frac{1}{2}}+\frac{\psi_{1}(0)}{\sqrt{3}}+0.327 \mathrm{fexp}\left(\frac{3 \psi_{2}}{2 \sigma_{0}}\right)
$$

Actually, the arowth of microvoids depends on the stress state. The plastic potential of spherical voids in the case of uniaxial tension is as

$$
{ }^{3} F_{1}=\frac{3 f}{(3-5 f)}\left[\frac{25 B^{2}}{16}+0.644 \sigma_{0} \cosh \left(\frac{3 B}{2 \sigma_{0}}\right)+0.009 \sigma_{0} \sinh \left(\frac{3 B}{2 \sigma_{0}}\right)\right]
$$

where $A=\psi_{i}(D), B=\psi_{2}(B), \sqrt{3}_{2}=\sigma_{0} / 3$ if the matrix is perfectly plastic medium. When the last term of eqn. (28) can be nealected, plastic potential is as

$$
{ }^{3} F=\left[J_{2}\left(\frac{\sigma}{\rho}\right)\right]^{\frac{1}{2}}+A / \sqrt{3}+\frac{3 f}{(3-5 f)}\left[\frac{25}{16} B^{2}+0.644 \sigma_{0} \cosh \left(\frac{3 B}{2 \sigma_{0}}\right)\right]
$$

\section{CONCLUSION AND DISCUSSION}

An anisotropic damade variable which has more qeneral sense was obtained by the associative method of macro-microscopical models. Present damage variable is associated with the size of microvoids. The state function taken in this paper consists of reversible and irreversible parts. The differential equation of state function was obtained. The olastic potential of spherical voids in the case of isotropic arowth was aiven. The plastic notential of anisotronic nrowth of spherical voids was also given in this paper.

It is noticed that the solution of differential equation for multivariable damae problem is not closed. The aeneral analytical solution of $\|$ should be given in next study.

\section{REFERENCE}

(1) Kachanov, L.M., Izv. Acad. Nauk SSSR, 0td. Techn. Nauk, No. $8,1958,26-31$

(2) Lemaitre, J. and Chaboche, J.L., J.Meca. Appl., Vol.2, No.3, $317-365,1978$.

(3) Rousselier, r., Proceeding of IUTAM Symposium, Fcance, June $2-5$, Ed.S. by Nemat-Nasser, 331-335, 1980. 
(4) Mclintock, F.A., J. of Appl. Mech., Vol.35, 363-371, 1968.

(5) Rice, J.R. and Tracey, J.M., J.Mech.Phys. Solids, Vol.17, 201-207, 1969. (6) Lemaitre, J., J. of Encin. Mater. and Tech., Vol.107,83-97,
l985.

(7) Eshelby, J.D. Droceedinas of the Royal Society, London; Series A, Vol.24l, 376-396, 1957.

(8) Tandon, F.P. and Wenn, F.J., J. of Appl. Mech., Vol.53, $511-518,1986$.

(9) Wenc, S.J., Int.J.Enain. Sci., Vol.22, No.7, 845-856, 1984. 